\title{
Orthogonality spaces allowing gradual transitions
}

\author{
Thomas Vetterlein \\ Department of Knowledge-Based Mathematical Systems \\ Johannes Kepler University, Altenberger Straße 69, 4040 Linz, Austria \\ Thomas.Vetterlein@jku. at
}

\begin{abstract}
We consider orthogonality spaces subject to the condition that gradual transitions between any two elements are possible. More precisely, given elements $e$ and $f$, we require a homomorphism from the unit circle to the automorphism group to exist such that one of these automorphisms maps $e$ to $f$, and any of these automorphisms leaves the elements orthogonal to $e$ and $f$ fixed. A natural example is the collection of one-dimensional subspaces of a real Hilbert space, endowed with the usual orthogonality relation. We show that the general case is closely related to this example.
\end{abstract}

Keywords: Orthogonality space, Real Hilbert space, Foundations of quantum mechanics.

\section{Introduction}

The notion of an orthogonality space was proposed in the 1960s by David Foulis and his collaborators [2]. In order to grasp the key features of the Hilbert space model of quantum physics, it is natural to focus on structures which can be considered as a simplification of that model and yet play a significant role from the physical point of view $[3,4]$. Trying out the limits of abstraction, one may restrict solely to the relation of orthogonality - to obtain the prototypical example of an orthogonality space. This way just one key aspect of physical modelling is taken into account, namely, the distinguishability of observation results.

We have tackled the problem of characterising the complex Hilbert spaces as special orthogonality spaces in our recent work [10, 11]. Our strategy was to make assumptions on the existence of certain automorphisms. In a sense, we described the complex unitary group as a particular transformation group, acting on a set endowed with a binary relation that is to be preserved. In the infinite-dimensional case [10], a characterisation on the basis of just a few natural assumptions turned out to be possible. The finite-dimensional case [11] has required a much deeper analysis and the assumptions are more involved.

In the present contribution we deal again with orthogonality spaces of finite rank. But we change the perspective as follows. Rather than focusing exclusively on a Hilbert space over the field of complex numbers, we investigate the consequences of a principle that seems to be of central relevance. The idea is simple: we require our orthogonality space to allow a gradual transition between any pair of its elements, realised by automorphisms that leave elements orthogonal to this pair unaffected. The precise formulation of this idea is admittedly not entirely free from technical subtleties, but all in all our condition reflects quite accurately the mentioned principle. Namely, let $e$ and $f$ be distinct elements of the orthogonality space. Then we suppose that a homomorphism from the abelian group of complex units to the group of automorphisms exists such that the latter act transitively on the closure of $e$ and $f$, one of them maps $e$ to an element orthogonal to $e$, and all elements orthogonal to two elements of the orbit of $e$ are mapped to themselves. We speak of an automorphism circle connecting $e$ and $f$.

Whether the orthogonality space should be thought of as the collection of atomic yes-no properties of a quantum physical system, or as the set of pure quantum states, is left open. In either case, the idea is that a smooth transition between two entities should be realisable by a family of automorphisms - in the sense just specified. We will see that the orthogonality space associated with a complex Hilbert space does not have the required property. The example that we may keep in mind is rather the real Hilbert space. Indeed, the main result of this contribution is the following: we show that an orthogonality space of finite rank that possesses enough automorphism circles to 
connect any two distinct elements arises from an orthomodular space, equipped with a positive definite symmetric bilinear form.

\section{Orthogonality spaces}

From the point of view of the discussions around the foundations of quantum mechanics, the following notion was first systematically studied in J. R. Dacey's Ph.D. Thesis [2].

Definition 2.1. An orthogonality space is a nonempty set $X$ equipped with a symmetric, irreflexive binary relation $\perp$, called the orthogonality relation. The rank of $(X, \perp)$ is the supremum of the number of mutual orthogonal elements.

The typical example is the collection of onedimensional subspaces of an inner-product space, endowed with the usual orthogonality relation, provided that the inner product is orthosymmetric (to ensure symmetry) and anisotropic (to ensure irreflexivity). The original motivating example is presumably a complex Hilbert space. But certainly, a (generalised) Hilbert space over any division ring gives rise to an example as well.

We will restrict our considerations to the case of a finite rank. The rank of an orthogonality space arising from an inner-product space of the indicated type is then simply its linear dimension.

We should remark that the central notion on which this work is based is actually quite unspecific. We can hardly claim that Definition 2.1 suggests by itself an application in the context of quantum physical modelling. Indeed, orthogonality spaces are essentially the same as (undirected) graphs, provided that the latter are defined as collections of two-element subsets of some universe. Hence their theory is, in principle, as general and rich as the whole area of graph theory.

Therefore, what matters is which structural properties we are going to consider in addition. Here, we are interested in investigating the effect of a postulate regarding automorphisms, which we have chosen with the intention of addressing a basic feature of quantum physical modelling.

An automorphism of an orthogonality space $(X, \perp)$ is a bijection $\varphi$ of $X$ such that $x \perp y$ if and only if $\varphi(x) \perp$ $\varphi(y)$. We denote by $\operatorname{Aut}(X)$ the automorphism group of $(X, \perp)$. We moreover denote by $\mathbb{T}$ the multiplicative group of complex units.

For $A \subseteq X$, we put $A^{\perp}=\{x \in X: x \perp a$ for all $a \in A\}$. The assignment $A \mapsto A^{\perp \perp}$ defines an operation on the power set of $X$, which is a closure operator. If $A^{\perp \perp}=A$ we say that $A$ is orthoclosed and we denote the set of all orthoclosed subsets of $X$ by $\mathcal{C}(X, \perp)$.

Lemma 2.2. $\mathcal{C}(X, \perp)$, partially ordered by settheoretical inclusion and equipped with the operation $\perp$, is a complete ortholattice.

Moreover, each automorphism of $(X, \perp)$ induces an automorphism of the ortholattice $\mathcal{C}(X, \perp)$.

\section{Automorphism circles}

We fix in this section an orthogonality space $(X, \perp)$ of finite rank.

We will moreover assume that $(X, \perp)$ fulfils the following property.

(A) For any distinct elements $e$ and $f$ of $X$, there is a group homomorphism $\kappa: \mathbb{T} \rightarrow \operatorname{Aut}(X)$ such that the following conditions are satisfied (we write $\kappa_{t}$, where $t \in \mathbb{R}$, for $\left.\kappa\left(e^{i t}\right)\right)$ :

$(\alpha)$ For any $g \in\{e, f\}^{\perp \perp}$, there is a $t \in \mathbb{R}$ such that $\kappa_{t}(e)=g$.

( $\beta$ ) We have $\kappa_{\frac{\pi}{2}}(e) \perp e$.

$(\gamma)$ Let $s \in \mathbb{R}$ be such that $\kappa_{s}(e) \neq e$. Then $\kappa_{t}(x)=x$ for any $x \perp e, \kappa_{s}(e)$ and $t \in \mathbb{R}$.

We will refer to $\kappa: \mathbb{T} \rightarrow \operatorname{Aut}(X)$, associated to some elements $e, f \in X$ according to condition (A), as an automorphism circle connecting $e$ and $f$.

Example 3.1. Let $H$ be a Hilbert space over $\mathbb{R}$. Let $P(H)$ be the associated projective space, endowed with the natural orthogonality relation. Then $(P(H), \perp)$ is an orthogonality space. Moreover, $(P(H), \perp)$ fulfils (A). Indeed, for any two linearly independent vectors $v, w \in H$, let $\kappa_{t}, t \in \mathbb{R}$, be the automorphism of $P(H)$ induced by the rotation by the angle $t$ in the (oriented) plane spanned by $v$ and $w$.

Lemma 3.2. Let $\kappa$ be an automorphism circle connecting the distinct elements $e, f \in X$. Then we have:

(i) The group $\kappa_{t}, t \in \mathbb{R}$, acts transitively on $\{e, f\}^{\perp \perp}$.

(ii) For each $t \in \mathbb{R}, \kappa_{t}(x)=x$ if $x \in\{e, f\}^{\perp}$.

(iii) For any $s, t \in \mathbb{R}$ such that $\kappa_{s}(e) \neq \kappa_{t}(e)$, we have $\{e, f\}^{\perp \perp}=\left\{\kappa_{s}(e), \kappa_{t}(e)\right\}^{\perp \perp}$

Proof. (ii) By condition ( $\alpha$ ) of (A) there is a $t \in \mathbb{R}$ such that $f=\kappa_{t}(e)$, hence this is a consequence of condition $(\gamma)$.

(i) In view of condition $(\alpha)$ of $(\mathrm{A})$, we only have to show that $\{e, f\}^{\perp \perp}$ is invariant under the action of $\kappa_{t}$ for any $t \in \mathbb{R}$. But by part (ii), each element of $\{e, f\}^{\perp}$ is left fixed by $\kappa_{t}$, hence $g \perp\{e, f\}^{\perp}$ implies $\kappa_{t}(g) \perp\{e, f\}^{\perp}$. 
(iii) Let $s, t \in \mathbb{R}$ as indicated. We show $\{e, f\}^{\perp}=$ $\left\{\kappa_{s}(e), \kappa_{t}(e)\right\}^{\perp}$. If $x \perp e, f$, then by part (ii) $x=$ $\kappa_{s}(x) \perp \kappa_{s}(e)$, and similarly we see that $x \perp \kappa_{t}(e)$. Conversely, if $x \perp \kappa_{s}(e), \kappa_{t}(e)$, then $\kappa_{-s}(x) \perp e, \kappa_{t-s}(e)$, hence by condition $(\gamma) \kappa_{r}\left(\kappa_{-s}(x)\right)=\kappa_{-s}(x)$, that is, $\kappa_{r}(x)=x$ for any $r \in \mathbb{R}$. We conclude $x=\kappa_{-s}(x) \perp e$ and by $(\alpha)$ it also follows that $x \perp f$.

We next note that $(\mathrm{A})$ implies the following transitivity property of $(X, \perp)$.

Lemma 3.3. Let $e, f \in X$. Then there is an automorphism $\varphi$ of $(X, \perp)$ such that $(\mathrm{i}) \varphi(e)=f$ and (ii) $\varphi(x)=x$ if $x \perp e, f$.

Proof. This is trivial in case $e=f$. Otherwise, the assertion follows from conditions $(\alpha)$ and $(\gamma)$ of $(\mathrm{A})$.

Lemma 3.4. Let $e, f \in X$ be distinct. Then there is an automorphism $\varphi$ of $(X, \perp)$ such that (i) $\varphi(e) \perp f$ and (ii) $\varphi(x)=x$ if either $x \perp e, f$ or $x \perp \varphi(e), f$.

Proof. Let $\kappa$ be an automorphism circle associated with $e, f$. Then $\kappa_{t}(e)=f$ for some $t \in \mathbb{R}$. Let $\varphi=\kappa_{t+\frac{\pi}{2}}$. Then $\varphi(e)=\kappa_{t}\left(\kappa_{\frac{\pi}{2}}(e)\right) \perp \kappa_{t}(e)=f$ by condition $(\beta)$. Moreover, the last assertion holds by Lemma 3.2 (ii),(iii).

The property stated in Lemma 3.4, called (F1) in [11], has quite strong consequences. We start with a technicality.

We call an orthogonality space irredundant if, for any pair of elements $x$ and $y,\{x\}^{\perp}=\{y\}^{\perp}$ implies $x=y$. In case this property fails, it is easy enough to switch to an irredundant one with essentially the same structure. In the present context, however, irredundancy comes anyhow as a consequence of (A).

Lemma 3.5. $(X, \perp)$ is irredundant.

Proof. This follows from Lemma 3.4; see [11, Lemma $2.6]$.

The next lemma establishes the key properties of $\mathcal{C}(X, \perp)$ used in the sequel. For lattice-theoretic notion, we refer to [5].

Lemma 3.6. $\mathcal{C}(X, \perp)$ is an atomistic modular ortholattice.

Moreover, the atoms of $\mathcal{C}(X, \perp)$ are exactly the singletons $\{x\}, x \in X$. In particular, the set of atoms of $\mathcal{C}(X, \perp)$, endowed with the inherited orthogonality relation, is isomorphic with $(X, \perp)$.

Proof. This is again a consequence of Lemma 3.4; see [11, Lemma 2.7, 2.11].
We conclude that the ortholattice $\mathcal{C}(X, \perp)$ has nearly all the properties that are typical for lattices of subspaces of an inner-product space. We address a further important property.

We say that $(X, \perp)$ is irreducible if $X$ is not the disjoint union of non-empty subsets $A, B \subseteq X$ such that $x \perp y$ for any $x \in A$ and $y \in B$.

Lemma 3.7. $(X, \perp)$ is irreducible.

Proof. Assume that $X=A \dot{\cup} B$, where $A, B \neq \varnothing$ and $x \perp y$ for any $x \in A$ and $y \in B$. Let $\kappa$ be an automorphism circle connecting some $e \in A$ and $f \in$ $B$. Then $\kappa_{\frac{\pi}{2}}(e) \perp e$ and $\kappa_{\frac{\pi}{2}}(e) \in\{e, f\}^{\perp \perp}=\{e\} \vee\{f\}$, hence $\kappa_{\frac{\pi}{2}}(e)=f$. Let $g \stackrel{\frac{\pi}{2}}{=} \kappa_{\frac{\pi}{4}}(e)$. Then $g \in B$ would imply $g \perp e$, hence $g=\kappa_{\frac{\pi}{4}}(e) \perp \kappa_{\frac{\pi}{4}}(g)=\kappa_{\frac{\pi}{2}}(e)=f$ and thus $g \in\{e, f\}^{\perp}$, in contradiction to the fact that $g \in\{e, f\}^{\perp \perp}$. Similarly, $g \in A$ would imply $g \perp f$ and $g=\kappa_{-\frac{\pi}{4}}(f) \perp \kappa_{-\frac{\pi}{4}}(g)=e$, again a contradiction.

Lemma 3.8. The lattice $\mathcal{C}(X, \perp)$ is irreducible.

Proof. Assume that $\mathcal{C}(X, \perp)$ is reducible. Then $\mathcal{C}(X, \perp)$ (as a lattice) is isomorphic to a direct product $L_{1} \times L_{2}$ of non-trivial (bounded) lattices $L_{1}$ and $L_{2}$. Moreover, $(a, 0) \perp(0, b)$ for any $a \in L_{1}$ and $b \in L_{2}$. It follows that the set of atoms of $\mathcal{C}(X, \perp)$ can be partitioned into two non-empty subsets such that any element of one set is orthogonal to any of the other one. In view of the second part of Lemma 3.6, we conclude that $(X, \perp)$ is not irreducible, in contradiction to Lemma 3.7.

We summarise that $\mathcal{C}(X, \perp)$ is an irreducible, atomistic modular ortholattice.

\section{Representation of orthogonality spaces in inner-product spaces}

In this section, $(X, \perp)$ will still be an orthogonality space of finite rank $n$ that fulfils (A). Our objective is its representation by means of an inner-product space.

We will exclude the case of rank $n \leqslant 3$ from our discussion, as the lattice-theoretic characterisation of linear spaces of low dimensions requires a different procedure; cf. Theorem 4.1 below. Accordingly, we assume that $n \geqslant 4$. We believe that from a physical point of view this restriction is inessential.

A $\star$-sfield will be a skew field (division ring) equipped with an involutorial anti-automorphism *. We use this practical notion to indicate both the possible noncommutativity and the presence of an involution. Let $H$ be a linear space over a $\star$-sfield $K$ and let $H$ be equipped with a hermitian form $(\cdot, \cdot): H \times H \rightarrow K$. 
This means that, for any $u, v, w \in H$ and $\alpha, \beta \in K$, we have

$$
\begin{aligned}
& (\alpha u+\beta v, w)=\alpha(u, w)+\beta(v, w), \\
& (w, \alpha u+\beta v)=(w, u) \alpha^{\star}+(w, v) \beta^{\star}, \\
& (u, v)=(v, u)^{\star} .
\end{aligned}
$$

Let the form moreover be anisotropic, that is, $(u, u)=$ 0 holds only if $u=0$. As usual, $u \perp v$ means $(u, v)=0$, where $u, v \in H$, and for a subspace $M$ of $H$ we let $M^{\perp}=\{u \in H: u \perp v$ for all $v \in M\}$. Moreover, we denote by $\mathcal{C}(H)=\left\{M \subseteq H: M^{\perp \perp}=M\right\}$ the set of closed subspaces. If for all $M \in \mathcal{C}(H)$ we have $H=M+M^{\perp}$, we refer to $H$ as an orthomodular space.

We write $[u]$ for the subspace spanned by a non-zero vector $u$ of an orthomodular space $H$, and we let $P(H)=\{[u]: u \in H \backslash\{0\}\}$. Then $P(H)$ becomes an irredundant orthogonality space.

Finite-dimensional orthomodular spaces can be characterised as follows by means of the ortholattice of their subspaces; see, e.g., [5].

Theorem 4.1. Let $H$ be a finite-dimensional orthomodular space. Then $\mathcal{C}(H)$ is an irreducible atomistic modular ortholattice of finite length.

Conversely, let $L$ be an irreducible atomistic modular ortholattice of finite length $\geqslant 4$. Then there is a $\star$-sfield $K$ and an orthomodular space $H$ over $K$ such that $L$ is isomorphic to $\mathcal{C}(H)$.

Let us recall Wigner's Theorem: Given an at least 3-dimensional orthomodular space $H$ and an at least 2-dimensional subspace $M$ of $H$, any automorphism $\varphi$ of $\mathcal{C}(H)$ such that $\varphi([u])=[u]$ for all $u \in M$ is induced by a unique unitary operator $U$ such that $\left.U\right|_{M}$ is the identity. See, e.g., [1].

Theorem 4.2. There is $a \star$-sfield $K$ and an orthomodular space $H$ over $K$ such that $\mathcal{C}(X, \perp)$ is isomorphic to $\mathcal{C}(H)$. In particular, $(X, \perp)$ is then isomorphic to $(P(H), \perp)$. The dimension of $H$ coincides with the rank of $(X, \perp)$. In addition, the inner product can be chosen such that each subspace of $H$ contains a unit vector.

Proof. The first assertion follows from Lemmas 3.6 and 3.8 by means of Theorem 4.1. The rank of $(X, \perp)$ is the maximal number of mutually orthogonal elements and coincides hence with the dimension of $H$.

Finally, the involution and the inner product can be modified with the effect that there is a subspace $[u]$ possessing a unit vector; see, e.g., [7]. By Lemma 3.3 and Wigner's Theorem, there is a unitary operator mapping $[u]$ to any given one-dimensional subspace. The last assertion follows.
Let $H$ be the orthomodular space over the $\star$-sfield $K$ representing $(X, \perp)$ according to Theorem 4.2. By assumption, $H$ is at least 4-dimensional.

Lemma 4.3. $K$ is commutative and the involution * is the identity.

Proof. Let $S$ be a two-dimensional subspace of $H$. Let $b_{1}, b_{2}$ be an orthogonal basis of $S$. Since each onedimensional subspace of $H$ contains a unit vector, we can assume that $b_{1}, b_{2}$ is in fact an orthonormal basis.

Let $\kappa$ be an automorphism circle connecting $\left[b_{1}\right]$ and $\left[b_{2}\right]$. For each $t \in \mathbb{R}, \kappa_{t}$ is by Wigner's Theorem induced by a unique unitary operator $U_{t}$ such that $\left.U_{t}\right|_{S^{\perp}}$ is the identity. Note that $U_{0}$ is the identity on $H$ and, due to the uniqueness part of Wigner's Theorem, $U_{s+t}=U_{s} U_{t}=U_{t} U_{s}$ for any $s, t \in \mathbb{R}$.

We shall identify the operators $U_{t}, t \in \mathbb{R}$, with their restriction to $S$. Then for each $t$ the operator is representable as a $2 \times 2$-matrix $U_{t}=\left(\begin{array}{ll}\alpha & \gamma \\ \beta & \delta\end{array}\right)$, where $\alpha \alpha^{\star}+\beta \beta^{\star}=\gamma \gamma^{\star}+\delta \delta^{\star}=1$ and $\alpha \gamma^{\star}+\beta \delta^{\star}=0$. In particular, let $T_{K}=\left\{\varepsilon \in K: \varepsilon \varepsilon^{\star}=1\right\}$ be the set of unit elements of $K$. By condition $(\beta)$ of $(\mathrm{A})$ we have $U_{\frac{\pi}{2}}\left(\left[b_{1}\right]\right)=\left[b_{2}\right]$ and consequently also $U_{\frac{\pi}{2}}\left(\left[b_{2}\right]\right)=\left[b_{1}\right]$. Hence $U_{\frac{\pi}{2}}=\left(\begin{array}{cc}0 & \varepsilon_{1} \\ \varepsilon_{2} & 0\end{array}\right)$ for some $\varepsilon_{1}, \varepsilon_{2} \in T_{K}$.

Because

$$
\begin{aligned}
\left(\begin{array}{ll}
\varepsilon_{2} \gamma & \varepsilon_{1} \alpha \\
\varepsilon_{2} \delta & \varepsilon_{1} \beta
\end{array}\right) & =\left(\begin{array}{cc}
\alpha & \gamma \\
\beta & \delta
\end{array}\right) \cdot\left(\begin{array}{cc}
0 & \varepsilon_{1} \\
\varepsilon_{2} & 0
\end{array}\right) \\
& =\left(\begin{array}{cc}
0 & \varepsilon_{1} \\
\varepsilon_{2} & 0
\end{array}\right) \cdot\left(\begin{array}{ll}
\alpha & \gamma \\
\beta & \delta
\end{array}\right)=\left(\begin{array}{ll}
\beta \varepsilon_{1} & \delta \varepsilon_{1} \\
\alpha \varepsilon_{2} & \gamma \varepsilon_{2}
\end{array}\right),
\end{aligned}
$$

each of the operators is of the form

$$
U_{t}=\left(\begin{array}{cc}
\alpha & \varepsilon_{1} \beta \varepsilon_{2}^{\star} \\
\beta & \varepsilon_{1} \alpha \varepsilon_{1}^{\star}
\end{array}\right)=\left(\begin{array}{cc}
\alpha & \varepsilon_{2}^{\star} \beta \varepsilon_{1} \\
\beta & \varepsilon_{2}^{\star} \alpha \varepsilon_{2}
\end{array}\right) .
$$

We next claim that $\beta^{-1} \alpha$, where $\left(\begin{array}{l}\alpha \\ \beta\end{array}\right)$ is the first column vector of some $U_{t}$ such that $\beta \neq 0$, ranges over all of $K$. Indeed, let $\xi \in K$. By Lemma 3.2 (i), there is an $t \in \mathbb{R}$ such that $U_{t}=\left(\begin{array}{ll}\alpha & \gamma \\ \beta & \delta\end{array}\right)$ maps $\left[e_{1}\right]$ to $\left[\xi e_{1}+e_{2}\right]$. Then $\beta \neq 0$ and $\left[\left(\begin{array}{l}\xi \\ 1\end{array}\right)\right]=\left[\left(\begin{array}{l}\alpha \\ \beta\end{array}\right)\right]$, thus the claim follows.

The orthogonality of the column vectors of the first matrix in (1) implies $\alpha \varepsilon_{2} \beta^{\star} \varepsilon_{1}^{\star}+\beta \varepsilon_{1} \alpha^{\star} \varepsilon_{1}^{\star}=0$ and hence $\left(\beta^{-1} \alpha\right)^{\star}=-\varepsilon_{1}^{\star} \beta^{-1} \alpha \varepsilon_{2}$. By the previous remark, we conclude $\xi^{\star}=-\varepsilon_{1}^{\star} \xi \varepsilon_{2}$ for any $\xi \in K$. From the case $\xi=1$ we see that $\varepsilon_{2}=-\varepsilon_{1}$. Let $\varepsilon=\varepsilon_{2}$. Then $\varepsilon \in T_{K}$ is such that

$$
\xi^{\star}=\varepsilon^{\star} \xi \varepsilon \text { for any } \xi \in K,
$$


and we conclude that for each $t \in \mathbb{R}$ there are $\alpha, \beta \in K$ such that

$$
U_{t}=\left(\begin{array}{cc}
\alpha & -\beta^{\star} \\
\beta & \alpha^{\star}
\end{array}\right)
$$

Let us furthermore consider the operator mapping $\left[e_{1}\right]$ to $\left[e_{1}+e_{2}\right]$, which is of the form $\left(\begin{array}{cc}\gamma & -\gamma^{\star} \\ \gamma & \gamma^{\star}\end{array}\right)$. Note that $2 \gamma \gamma^{\star}=1$ and hence $\gamma^{\star}=\frac{1}{2} \gamma^{-1}$. This operator commutes with any operator $(2)$, hence we have

$$
\begin{aligned}
\left(\begin{array}{cc}
\tilde{\alpha} & -\tilde{\beta}^{\star} \\
\tilde{\beta} & \tilde{\alpha}^{\star}
\end{array}\right) & =\left(\begin{array}{cc}
\gamma & -\gamma^{\star} \\
\gamma & \gamma^{\star}
\end{array}\right) \cdot\left(\begin{array}{cc}
\alpha & -\beta^{\star} \\
\beta & \alpha^{\star}
\end{array}\right) \\
& =\left(\begin{array}{cc}
\alpha & -\beta^{\star} \\
\beta & \alpha^{\star}
\end{array}\right) \cdot\left(\begin{array}{cc}
\gamma & -\gamma^{\star} \\
\gamma & \gamma^{\star}
\end{array}\right) .
\end{aligned}
$$

This means

$$
\begin{aligned}
& \alpha \gamma-\beta \gamma^{\star}=\gamma \alpha-\gamma \beta^{\star}=\tilde{\alpha}, \\
& \alpha \gamma+\beta \gamma^{\star}=\gamma \beta+\gamma \alpha^{\star}=\tilde{\beta}, \\
& \gamma \alpha+\gamma^{\star} \beta=\alpha^{\star} \gamma+\beta \gamma=\tilde{\beta}, \\
& \gamma \alpha-\gamma^{\star} \beta=\alpha \gamma-\beta^{\star} \gamma=\tilde{\alpha} .
\end{aligned}
$$

Consequently, $2 \alpha \gamma=2 \gamma \alpha=\tilde{\alpha}+\tilde{\beta}$ and $2 \beta \gamma^{\star}=2 \gamma^{\star} \beta=$ $\tilde{\beta}-\tilde{\alpha}$. Hence $\gamma$ commutes with $\alpha$ and $\beta$, and we conclude that $\gamma$ is in the centre of $K$. In particular, $\gamma^{\star}=\gamma$. Furthermore, we have $\left(\alpha+\beta+\alpha^{\star}-\beta^{\star}\right) \gamma=$ $\tilde{\alpha}+\tilde{\beta}=2 \alpha \gamma$ and $\left(\beta-\alpha+\alpha^{\star}+\beta^{\star}\right) \gamma=\tilde{\beta}-\tilde{\alpha}=2 \beta \gamma$. It follows $\alpha^{\star}-\beta^{\star}=\alpha-\beta$ and $\alpha^{\star}+\beta^{\star}=\alpha+\beta$, that is, $\alpha=\alpha^{\star}$ and $\beta=\beta^{\star}$.

Since $\alpha=\alpha^{\star}=\varepsilon^{\star} \alpha \varepsilon$, we have $\alpha \varepsilon=\varepsilon \alpha$, and similarly we see that $\beta \varepsilon=\varepsilon \beta$. Hence $\left(\beta^{-1} \alpha\right)^{\star}=\varepsilon^{\star} \beta^{-1} \alpha \varepsilon=$ $\beta^{-1} \alpha$, provided that $\beta \neq 0$. We conclude $\xi^{\star}=\xi$ for any $\xi \in K$. That is, the involution is the identity, and the $\star$-sfield is commutative.

Thus we have shown that the space $H$, which represents the orthogonality space $(X, \perp)$, is an orthomodular space over a field $K$, and the inner product is bilinear and symmetric.

We finally show that $K$ is orderable, or formally real, and that, whatever the order is chosen, the inner product is positive definite. For the field $K$ to be ordered means that $K$ is equipped with a total order such that (i) $\alpha \leqslant \beta$ implies $\alpha+\gamma \leqslant \beta+\gamma$ and (ii) $\alpha, \beta \geqslant 0$ implies $\alpha \beta \geqslant 0$.

Consider the set of sums of squares in $K$, that is,

$$
S_{K}=\left\{\alpha_{1}^{2}+\ldots+\alpha_{k}^{2}: \alpha_{1}, \ldots, \alpha_{k} \in K, k \geqslant 0\right\} .
$$

W.r.t. any order on $K, S_{K}$ consists solely of positive elements. The strategy to show that $K$ is orderable is to verify $S_{K} \cap-S_{K}=\{0\}$; it then follows that $S_{K}$ can be extended to a positive cone determining an order that makes $K$ into an ordered field. We will use this argument to prove our final lemma.

We refer to [9] for further information on the topic of fields and orderings.

Lemma 4.4. $K$ is a formally real field. W.r.t. any order on $K$, the hermitian form on $E$ is positive definite.

Proof. Let $\alpha_{1}, \ldots, \alpha_{k} \in K, k \geqslant 2$, such that $\alpha_{1}^{2}+\ldots+$ $\alpha_{k}^{2}=0$. Our aim is to show that $\alpha_{1}, \ldots, \alpha_{k}=0$. It will then follow that $S_{K} \cap-S_{K}=\{0\}$ and hence that $K$ is formally real $[9, \S 1]$.

Let $b_{1}, b_{2}$ be orthogonal unit vectors of $H$. Assume first that $k=2$. Then $\left(\alpha_{1} b_{1}+\alpha_{2} b_{2}, \alpha_{1} b_{1}+\alpha_{2} b_{2}\right)=$ $\alpha_{1}^{2}+\alpha_{2}^{2}=0$ implies $\alpha_{1} b_{1}+\alpha_{2} b_{2}=0$ and thus $\alpha_{1}=$ $\alpha_{2}=0$.

Let now $k \geqslant 3$ be arbitrary. Let $v=\alpha_{1} b_{1}+\alpha_{2} b_{2}$. By Lemma 3.3, there is a unitary operator $U$ such that $U([v])=\left[b_{1}\right]$. Then $U(v)=\beta_{1} b_{1}$ for some $\beta_{1} \in K$, and we have $\beta_{1}^{2}=\alpha_{1}^{2}+\alpha_{2}^{2}$. Arguing repeatedly in this way, we see that there is a $\beta_{i}$ such that $\beta_{i}^{2}=\beta_{i-1}^{2}+\alpha_{i+1}^{2}$, $i=2, \ldots, k-1$. But then, $0=\alpha_{1}^{2}+\ldots+\alpha_{k}^{2}=\beta_{k-1}^{2}=$ $\beta_{k-2}^{2}+\alpha_{k}^{2}$ implies $\beta_{k-2}=\alpha_{k}=0$, and we conclude successively in the same way that $\alpha_{i}=0$ for all $i$.

For the second assertion, we recall that, for any $v \in$ $H \backslash\{0\}$, there is a unit vector $b \in H$ and an $\alpha \in K$ such that $v=\alpha b$. But then $(v, v)=(\alpha b, \alpha b)=\alpha^{2}>0$.

We summarise what we have shown.

Theorem 4.5. Let $(X, \perp)$ an orthogonality space fulfilling (A). Then there is an ordered field $K$ and an orthomodular space $H$ over $K$, based on a positivedefinite symmetric bilinear form, such that $(X, \perp)$ is isomorphic to $(P(H), \perp)$.

\section{Conclusion}

Approaches to reconstruct the basic model of quantum mechanics, the complex Hilbert space, from physically plausible principles often exploit the fact that there are smooth transitions between any two (pure) states of a quantum physical system. The present work investigates the question what we can conclude from this fact alone. Transitions are understood as automorphisms, and we have chosen a minimal structure to which this notion refers. An orthogonality space consists of not more than the relation after which it is named.

We were in this way not led to Hilbert spaces over $\mathbb{C}$. It was actually clear from the outset that the chosen postulate fits instead quite well to the case of a real Hilbert space. We have in fact shown that any orthogonality space of the considered kind arises from 
a generalised Hilbert space (an orthomodular space) over an ordered field.

We believe that this study could nonetheless help to facilitate understanding of the complex case as well. We have shown in our previous works that we may identify the complex Hilbert space with orthogonality spaces in which certain automorphisms are assumed to exist. We are in that case faced with the fact that each single element of the orthogonality space gives rise to a group of automorphisms, similarly to our automorphism circles. However, these automorphisms are difficult to "see" in the abstract setting of an orthogonality space. To make the relationship between the automorphisms associated with single elements and symmetries of the whole space more clear, the detour via the real Hilbert space might make sense. After all, real Hilbert spaces endowed with a complex structure lead to the model of the desired type.

Having said this, it clearly follows that it would be desirable to continue the present line of investigation so as to actually achieve a characterisation of the real Hilbert spaces. We conjecture that to this end, additional postulates or additional structure would be necessary. Two possibilities could be considered. Holland has shown in his paper [6] that linear spaces over (Baer) ordered $\star$-sfields equipped with a positive definite hermitian form possess a particular kind of quotient, leading to a space over (a subfield of) a classical $\star$-sfield. We could make use of the fact in analogy to our procedure in [11], requiring that the orthogonality space possesses no non-trivial quotients compatible with the automorphism circles. A second way involves topology. One could consider topological orthogonality spaces, similar to Wilce's topological test spaces [12], to the effect that also the representing linear space can be endowed with a suitable topology. A well-known result of Pontrjagin [8] might then be useful, according to which a locally compact, connected topology on a $\star$-sfield implies that the latter is among the three classical ones.

\section{References}

[1] G. Chevalier, Wigner's theorem and its generalizations, in: Handbook of quantum logic and quantum structure. Quantum structures, Amsterdam: Elsevier, 2007, pp. 429-475.

[2] J. R. Dacey, Orthomodular spaces, Ph.D. thesis, University of Massachusetts, Amherst, USA (1968).

[3] K. Engesser, D. M. Gabbay, D. Lehmann (Eds.), Handbook of quantum logic and quantum structure. Quantum structures, Amsterdam: Elsevier, 2007.
[4] K. Engesser, D. M. Gabbay, D. Lehmann (Eds.), Handbook of quantum logic and quantum structures. Quantum logic, Amsterdam: Elsevier/North-Holland, 2009.

[5] S. M. F. Maeda, Theory of symmetric lattices, Springer-Verlag, Berlin - Heidelberg - New York, 1970.

[6] S. S. Holland, «-valuations and ordered $\star$-fields, Trans. Am. Math. Soc. 262 (1980) 219-243.

[7] S. S. Holland, Orthomodularity in infinite dimensions; a theorem of M. Solèr, Bull. Am. Math. Soc., New Ser. 32 (1995) 205-234.

[8] L. Pontrjagin, Über stetige algebraische Körper, Ann. Math. (2) 33 (1932) 163-174.

[9] A. Prestel, Lectures on formally real fields, Springer-Verlag, Berlin - Heidelberg - New York Tokio, 1984.

[10] T. Vetterlein, Orthogonality spaces arising from infinite-dimensional complex Hilbert spaces, submitted; available on http://www.flll.jku.at/sites/ default/files/u24/Orthogonality-spaces-ofinfinite-rank.pdf.

[11] T. Vetterlein, Orthogonality spaces of finite rank and the complex Hilbert spaces, submitted; available on http://www.flll.jku.at/sites/default/files/ u24/Orthogonality-spaces-of-finite-rank.pdf.

[12] A. Wilce, Topological test spaces, Int. J. Theor. Phys. 44 (2005) 1227-1238. 